\title{
A REMARK ON FINITELY GENERATED MODULES
}

\author{
TADASI NAKAYAMA
}

Theorem 5 of Azumaya's recent article ${ }^{1:}$ can be formulated in the following generalized form:

I. Let $R$ be a ring. Let $m$ be a finitely generated right-module of $R$ such that $m R=m$. Assume that $\mathrm{m}=u_{3} r+u_{2} r+\ldots+u_{m} r$ for every generating system $u_{1}, u_{2}, \ldots, u_{m}$ of $m$ and for every maximal right-ideal $r$ of $R$. Then $m=0$.

For the proof, we first consider the case where $R$ possesses a unit element 1. Then the assertion can be proved quite similarly as in Azumaya, 1. c. Let namely $u_{1}, u_{2}, \ldots, u_{m}$ be any finite generating system of $\mathrm{m} ; \mathrm{m}=u_{1} R+u_{2} R+\ldots$ $+u_{m} R$. Let $r_{n}$ be the right-ideal of $R$ consisting of all elements $x$ of $R$ such that

$$
u_{1} x \in u_{2} R+\ldots+u_{m} R .
$$

Suppose $r_{0} \neq R$. There exists a maximal right-ideal $r$ which contains $r_{0}$, and we have $m=u_{1} r+u_{2} r+\ldots+u_{m} r$, whence $=u_{1} r+u_{2} R+\ldots+u_{m} R$ much the more, by our assumption. There is an element $a$ in $r$ such that $1-a \in r_{1} \leqq r$. which is a contradiction. Hence necessarily $r_{0}=R$ and $\mathrm{m}=u_{2} R+\ldots+u_{m} R$. Now the assertion can be proved by an induction with respect to the minimal number of generating elements.

Let next $R$ be general. Let $R^{*}$ be the ring which is as module a direct sum of $R$ and the ring of rational integers and in which $1 x=x 1=x(x \in R)$. If $r^{*}$ is a maximal right-ideal of $R^{*}$, then $r^{*} \cap R$ is either $R$ or a maximal right-ideal of $R$. Thus $m=u_{1} r^{*}+u_{2} r^{*}+\ldots+u_{m} r^{*}$, much the more, and the assertion can be reduced to the above case.

It is perhaps of interest to observe that from this generalization Jacobson's theorem ${ }^{2}$ may be derived:

Received May 15, 1951.

1) G. Azumaya, On maximally central algebras, Nagoya Math. J. 2 (1951).

$\therefore$ N. Jacobson, The radical and semi-simplicity for arbitrary rings, Amer. J. Math. 67 (1945), Theorem 10; the present formulation is in 1), l. c. 
II. Let $R$ be a ring and $N$ be its radical. If $\mathfrak{m}$ is a finitely generated rightmodule of $R$ and if $m=m N$, then $m=0$.

For, the radical $N^{*}$ of $R^{*}$, as above, contains (coincides with, as a matter of fact) $N$. Hence $\mathrm{m} N^{*}=\mathrm{m}$. Then $\mathrm{m}=u_{1} N^{*}+u_{\Omega} N^{*}+\ldots+u_{m} N^{*}$ for any generating system $u_{1}, u_{2}, \ldots, u_{m}$. Since every maximal right.ideal of $R^{*}$ contains $N^{*}$, the assertion is an immediate consequence of $\mathrm{I}$.

Next we want to observe:

III. In I we may restrict ourselves to those maximal right-ideals $r$ which contain the radical $N$.

We consider namely the residue-module $m / m N$ and find, by virtue of $I$, $m=m N$. Then $m=0$ because of II. (It would also be possible to prove III directly.)

Now, it is clear that the radical is the largest two-sided ideal possessing the property of II. Namely:

IV. If $M$ is a two-sided ideal and if $m=0$ is the only finitely generated module with $\mathrm{m}=\mathfrak{m} M$, then $M \cong N$.

For, if $M 末 N$, there exists a maximal right-ideal $r$ with left modulo-unit $a$ such that $r=M$. The right-module $\mathfrak{n}=R / r$ of $R$, generated by $a(\bmod r)$, satisfies $\mathrm{m}=\mathrm{m} M$, since $a M \equiv M \equiv R \bmod r$.

Similary, the family of right-ideals of III gives the "natural boundary," i.e.:

V. A family of right-ideals $\left\{r^{\prime}\right\}$ of $R$ possesses the property of I (with $\left\{r^{\prime}\right\}$ in place of $\{\boldsymbol{r}\}$ ) if and only if for every maximal right-ideal $r$ with left modulounit there exists an $r^{\prime}$ in the family such that $r^{\prime} \leqq r$.

It is evident that if this is the case then $\left\{r^{\prime}\right\}$ possesses the required property. On the other hand, if there exists a maximal right-ideal $r$ with left modulo-unit $a$ such that $r$ 決 $r^{\prime}$ for every $r^{\prime} \in\left\{r^{\prime}\right\}$, then $m=R / r$ satisfies $m=m r^{\prime}$ for every $r^{\prime}$, as in IV.

Needless to say that I, II, III and IV are special cases of V. Further, we may replace "finitely generated module" by ("cyclic module," or) "minimal module" in V (and IV) (and then the statement includes also a well known characterization of the radical).

\section{Nagoya University}

and

University of Illinois 